\title{
DEMOSAICING OF IMAGES FOR BAYER CFA USING PROJECTION
}

\section{ALGORITHM}

\author{
R.Niruban ${ }^{1}$, T.Sree Renga Raja ${ }^{2}$, R.Deepa ${ }^{3}$ \\ ${ }^{1}$ Assistant Professor, Electronics and Communcation Engineeering, Anand Institute of Higher Technoloy, TN ,India \\ ${ }^{2}$ Associate Professor,Electrical and Electronics Department, Anna university,Thiruchirapalli,,TamilNadu,India \\ ${ }^{3}$ Assistant professorComputer scienc and engineering,Dr.K.Vasudevan college of engineering and \\ Technology,TamilNadu,India,
}

\begin{abstract}
Typical consumer digital camera sense only one out of three components per image pixel because of increase in size and cost of sensor used in camera. An effective demosaicing is presented to restore the missing pixels of image captured from single sensor cameras. To eliminate most of color artifacts in edge region, Edge based demosaicing algorithm is to interpolate missing green sample followed by interpolate red and blue samples. Many demosaicing algorithms find edges in horizontal and vertical directions, which are not suitable for other directions. Before using the algorithm Gaussian filter is used for edge enhancement and smoothing of image. This proposed algorithm will be compared with other existing algorithms using PSNR measure.
\end{abstract}

Key Words: Demosaiking, Gaussian filtering, Iterative demosaicing, Refining

\section{INTRODUCTION}

Digital devices include modern computers, mobile phones, laptop and other electronic devices are inbuilt with digital cameras, because of small in size and cost-effective. Many peoples are choosing devices like mobile phone to capture image instead of using high digital cameras, the reason is low cost and also more convenient.

Single sensor used in this camera is CCD (or) CMOS sensor and the filter used is a color filter array. CMOS sensors required relatively low power where as CCD sensors [4-7] respond in low light condition also. Therefore both this sensors deliver high quality image at the same time consumer friendly. Filter used with single sensor to capture the image called CFA(Color Filter Array) but it samples only one color in an image. There are different CFA patterns but the commonly used one is a Bayer pattern which composed of $50 \%$ of green, $25 \%$ of red and $25 \%$ of blue. It will sample more green values because of human eye is more sensitive to green compare to red and blue. Remaining color samples missing in each pixel in bayer pattern are interpolated by using the method called demosaicing (or) color filter array interpolation.

There are many demosaicing algorithms used for interpolating missing color samples. First one is the bilinear interpolation which interpolates red value in non red pixel and interpolates blue value in non blue pixel, similarly for green by using two or four adjacent red and blue pixels for compute red and blue samples respectively. Bilinear interpolation does not interpolate in edge region and cause zipper effect in edge regions. Cubic interpolation [2] is similar to [1] and at the same time to interpolate R,G,B it take the four pixels that are closest to the diagonal corners and average their value to produce the middle pixel. Bicubic interpolation, in contrast takes not only the four closest diagonal pixels, but their closest pixels as well, for a total of 16 pixels. This method is efficient but introduces large errors in edge regions.

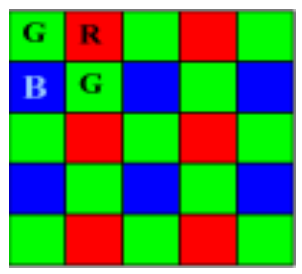

Fig 1[11]: Bayer Pattern

The above described methods are non adaptive algorithms. Now we discuss about the adaptive methods. In non linear method also called adaptive method green sample is interpolated first where red and green is interpolated by using green.

In smooth hue transition method, cross correlation takes place, it removes the false color during the demosaicing process, but it suffers from color artifacts. It uses constant color difference method between red, green and blue pixels. Another non linear method used is edge gradient based interpolation method. Gradient based method [13] is nothing but extensive of adaptive color plane method (ACPI)[17]. In ACPI we consider only two directions but in this method two more directions are considered to calculate gradient of missing $\mathrm{G}$ samples. Weight is determined for each direction using known $\mathrm{R}$ or $\mathrm{B}$ and $\mathrm{G}$ values. 
In this section, a method to determine green by using four direction gradient is developed and also implement the algorithm which defines feasible sets based on obtained colors in each pixel and experience the inter channel correlation between the channels. Our algorithm is compared with various existing demosaicing algorithm by using PSNR measurement. Section 2 presents the preprocessing process. Section 3 declares the benefits and purpose of this algorithm. The comparison of other existing algorithm with our algorithm is given in section 4 . Conclusion is provided in section 5 .

\section{PREPROCESSING}

The two step pre processing procedure is used in this paper. Image must be suitable for further processing and matlab environment so preprocessing process takes place. Here Gaussian filter is used for preprocessing which enhances the image quality and also used for image restoration. Gaussian filtering is used to remove noise and weight gives high significance to the pixels near edge.

2.1 Gaussian Filter: The impulse response function of Gaussian filter is the function of Gaussian filter. Mathematically, Gaussian filters alternating the input value by using convolution with impulse response. The Gaussian filter is a continuous and is not a discrete function. The cut off frequency $\mathrm{Fc}$ of the Gaussian filter is calculated as the ratio between square root of variance and sample rate Fs.

The Gaussian filter is defined by the equation

$$
g(x)=\frac{1}{\sqrt{2 \pi} \sigma} e^{\frac{x^{2}}{2 \sigma^{2}}}
$$

The impulse response function of 1D Gaussian filter is given as

$$
g(x)=\frac{1}{\sqrt{2 \pi} \sigma} e^{\frac{-\sigma^{2} u^{2}}{2}}
$$

In this preprocessing setup, CFA image is filtered and enhanced by Gaussian filter results in error reduction smoothening of image and it is passed through Gaussian filter for further processing.

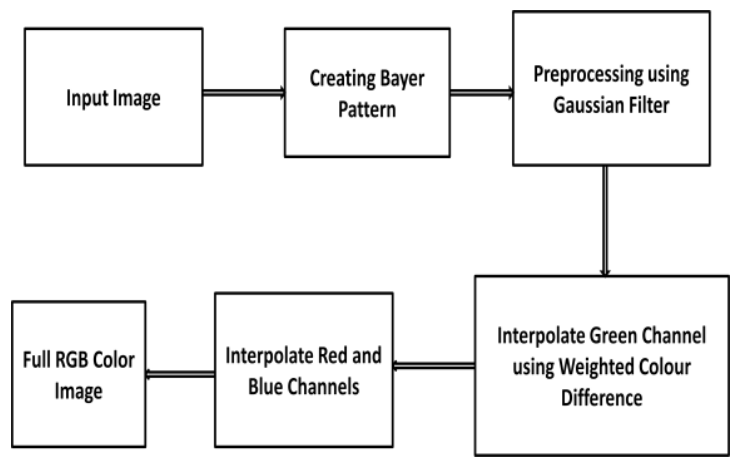

Fig 2: BLOCK DIAGRAM

\section{DEMOSAICING USING WEIGHTED EDGE METHOD}

In natural images, there is a high correlation between red, green and blue channels. The correlation between these three samples is same in both texture and edge region. While using camera having CFA in which luminance called green is sampled at higher rate than chrominance (red, blue pixels). Therefore performance of red and blue pixels is little poor as compare to the green and at the same time aliasing effect is high in red and blue samples. This paper suggests a new demosaicing algorithm with feasible sets used for remove color artifacts and aliasing in high frequency regions include edges. It sense that interpolated missing red and blue is observed and reconstruct them by using cross channel correlation which is projecting onto feasible sets.

Before applying this constraint set, decompose the channel by using filter bank structure. In filter bank $\mathrm{H}_{0}(\mathrm{z})$ is a low pass filter and $\mathrm{H}_{1}(\mathrm{z})$ is a high pass filter. Section 3(A) defines how the missing green in every pixel is interpolated using edge gradient based interpolation method. In section 3(B) declares interpolation of $\mathrm{R}$ and $\mathrm{B}$ using cubic interpolation. Section $\mathrm{C}$ defines feasible sets used by the proposed algorithm.

\section{DEMOSAICING USING WEIGHTED EDGE METHOD}

Image consists of three color planes. Red, Blue, and Green plane. In our proposed algorithm, the missing color plane is interpolated using remaining color planes. Based on this method, missing green is determined first later missing red and blue color samples in each pixel is determined. Here original image pixel value is $R, G, B$ and $R{ }^{*}, G^{*}, B^{*}$ are interpolated pixel value.

In order to find missing green in non green pixels, determine weight in four sides of required pixel then the color difference in four sides of pixel is determined by neighboring pixel value. The weight assign to color difference is used to estimate the missing green pixel. Finally calculate two color difference planes $\mathrm{Kr}$ and $\mathrm{Kb}$. In first step, we have only to determine the gradient for the red and blue channel. The gradient is the green channel so we consider on green pixel that is actually around the red pixel. Likewise interpolate green pixel from the blue component. Let us take fig-3 to explain how to estimate $G$ channel value at R33.Before performing interpolation for $G$ channel determine the gradient for red and blue pixel.

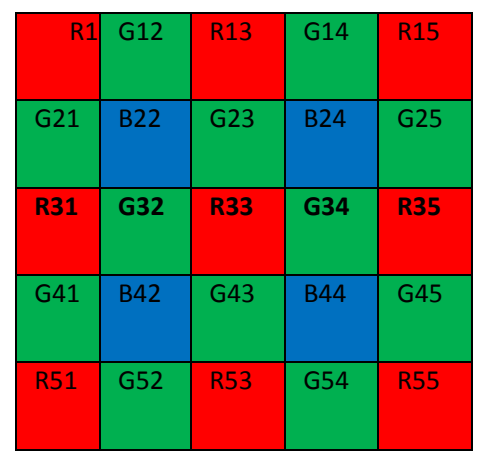

Fig -1: 5x5 Bayer pattern 
As shown in Fig (3), to obtain the G33 value at the R33 pixel first to calculate the gradient along the four adjacent directions as follows

$$
\begin{gathered}
\alpha_{2,3}=\left|\mathrm{R}_{1,3}-\mathrm{R}_{3,3}\right|+\left|\mathrm{G}_{2,3}-\mathrm{G}_{4,3}\right| \\
\alpha_{4,3}=\left|\mathrm{R}_{5,3}-\mathrm{R}_{3,3}\right|+\left|\mathrm{G}_{2,3}-\mathrm{G}_{4,3}\right| \\
\alpha_{3,2}=\left|\mathrm{R}_{3,1}-\mathrm{R}_{3,3}\right|+\left|\mathrm{G}_{3,2}-\mathrm{G}_{3,4}\right| \\
\alpha_{3,4}=\left|\mathrm{R}_{3,5}-\mathrm{R}_{3,3}\right|+\left|\mathrm{G}_{3,2}-\mathrm{G}_{3,4}\right|
\end{gathered}
$$

The Four adjacent color differences is calculated as follows

$\mathrm{K} 2,3=\mathrm{G}_{2,3}-1 / 2(\mathrm{R} 1,3+\mathrm{R} 3,3)$

$$
\begin{aligned}
& \mathrm{K} 4,3=\mathrm{G}_{4,3}-1 / 2(\mathrm{R} 5,3+\mathrm{R} 3,3) \\
& \mathrm{K} 3,2=\mathrm{G}_{3,2}-1 / 2\left(\mathrm{R}_{3,1}+\mathrm{R} 3,3\right) \\
& \mathrm{K} 3,4=\mathrm{G}_{3,4}-1 / 2\left(\mathrm{R}_{3,5}+\mathrm{R}_{3,3}\right)
\end{aligned}
$$

The weights as defined in equation (1) are then assigned to the four adjacent color difference values $\mathrm{K}$, as defined In equation (2), The estimating k3,3 is calculated as follows

$$
\begin{aligned}
& K 1=\left(K_{2,3}\right) /\left(1+\alpha_{2,3}\right) \\
& K 2=\left(K_{4,3}\right) /\left(1+\alpha_{4,3}\right) \\
& K 3=\left(K_{3,2}\right) /\left(1+\alpha_{3,2}\right) \\
& K 4=\left(K_{3,4}\right) /\left(1+\alpha_{3,4}\right)
\end{aligned}
$$

$K_{3,3}=(K 1+K 2+K 3+K 4) /((1+\alpha 2,3)+(1+\alpha 4,3)+(1+\alpha 3,2)+(1+\alpha 3,4))$

Then the Missing Green pixel value in red CFA components is calculated as follows

$\mathrm{G}^{*} 3,3=\mathrm{R} 3,3+\mathrm{K}_{3,3}$

Similarly the green pixel value is calculated in blue cfa components.

\section{INTERPOLATING MISSING COMPONENT AT GREEN}

After interpolating all green color planes of the image, interpolate missing red and blue samples in all green position. For interpolate red and blue at green position consider also the green components which is interpolated from red and blue. Figure.4 (a) shows the green CFA sample located in the red green row and blue green column to interpolate red and blue horizontally and vertically located pixels.

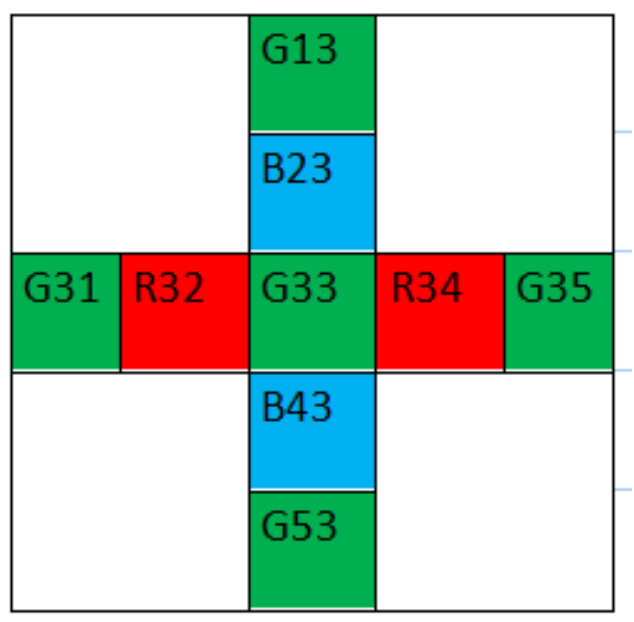

Fig 4(a): Interpolate missing $\mathrm{R}$ in $\mathrm{G}$

$$
\begin{aligned}
& R_{3,3}^{*}=G_{3,3}+\left[R_{3,2}-G_{3,2}^{*}+R_{3,4}-G_{3,4}^{*}\right] / 2 \\
& B_{3,3}^{*}=G_{3,3}+\left[B_{2,3}-G^{*}{ }_{2,3}+B_{4,3}-G^{*}{ }_{4,3}\right] / 2
\end{aligned}
$$

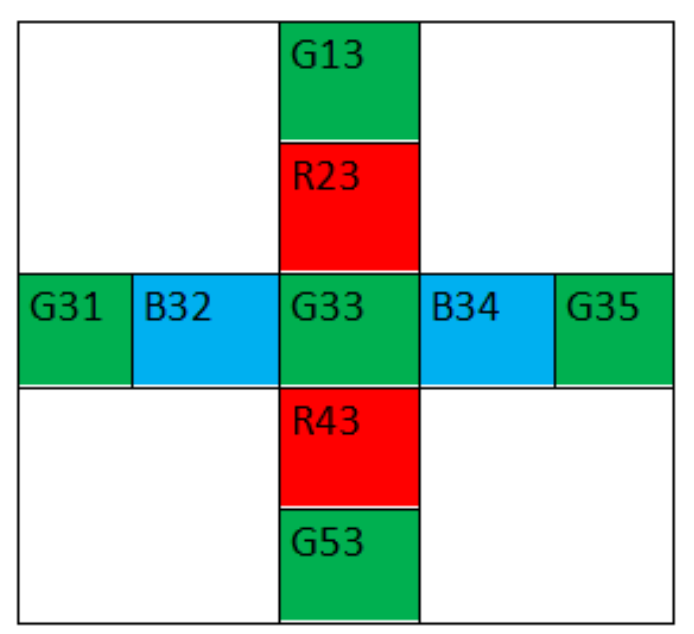

Fig 4(b): Interpolate missing B in G

Figure.4(b) shows the green CFA sample located in the red green column and blue green row to interpolate red and blue vertically and horizontally located pixels.

$$
\begin{aligned}
& R_{3,3}^{*}=G_{3,3}+\left[R_{2,3}-G^{*}{ }_{2,3}+R_{4,3}-G^{*}{ }_{4,3}\right] / 2 \\
& B_{3,3}^{*}=G_{3,3}+\left[B_{3,2}-G_{3,2}^{*}+B_{3,4}-G^{*}{ }_{3,4}\right] / 2
\end{aligned}
$$

\section{INTERPOLATE MISSING R(B) COMPONENT AT B(R) SAMPLING POSITION}

Finally the missing red components at the blue sampling position are interpolated. Fig. 5, shows the possible cases where pixel of interest lies in the centre of a $5 \times 5$ window. Here red pixels are interpolated by using bilinear interpolation method over color difference. For the case in Fig.4 (b), the centre missing red sample, $R^{*}{ }_{i, j}$, is interpolated by

$$
R_{i, j}^{*}=G_{i, j}+\frac{1}{4} \sum_{m=\mp 1} \sum_{n=\mp 1}\left(R_{i+m, j+n}-G_{i+m, j+n}\right)
$$

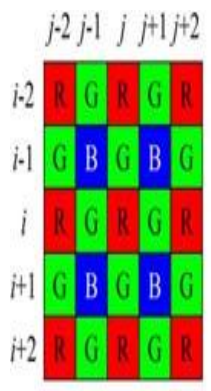

(a)

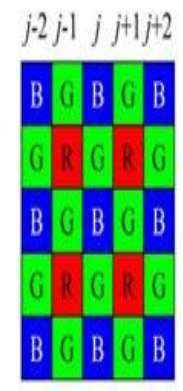

(b)

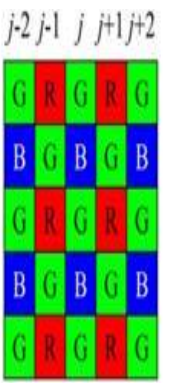

(c)

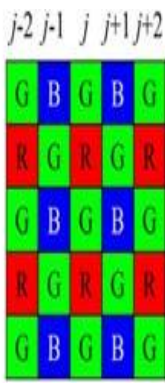

(d)
Fig 5[17]: Example of CFA configuration 
As for the case in Fig. 5(a), the centre missing blue sample, $R_{i, j}^{*}$, can also be obtained with (4) by replacing the red estimates with the corresponding blue estimates in (5). At last, the final full color image can be obtained.

The missing blue component in red is interpolated by

$$
B^{*}{ }_{i, j}=G_{i, j}+\frac{1}{4} \sum_{m=\mp 1} \sum_{n=\mp 1}\left(B_{i+m, j+n}-G_{i+m, j+n}\right)
$$

\section{FEASIBLE SETS}

Plan a conceptual reconstruction process produce a solution which is accurate with the details appear from detected data or initial knowledge about the solution. For demosaicing approach in our method, two types of feasible sets, one based on the perceive data and other depend on cross-channel correlation. The missing color samples which are interpolated must be acting in same way with color samples already present in CFA of digital camera. We declared $D\left(t_{1}, t_{2}\right)$ as this observed data contains $R, G, B$ placed according to Bayer pattern. $R^{*}, G^{*}, B{ }^{*}$ are set of pixel point. $\left(t_{1}, t_{2}\right)$ which defines the pixel point have samples of red, green and blue samples respectively.

The equation for determined feasible set is as follows:

$$
\mathrm{F}_{\mathrm{o}}=\left\{\mathrm{X}\left(\mathrm{t}_{1}, \mathrm{t}_{2}\right)=\mathrm{D}\left(\mathrm{t}_{1}, \mathrm{t}_{2}\right) \forall\left(\mathrm{t}_{1}, \mathrm{t}_{2}\right) \in \Lambda_{\mathrm{s}},(\mathrm{X})=\mathrm{R}, \mathrm{G}, \mathrm{B}\right\}
$$

Where $\mathrm{X}$ is a common symbol for interpolating missing color samples which may be R for red channel, G for Green and B for blue respectively.

The first observed data obtained using constraint set is not enough to define constraint sets of high frequency sub bands similar to luminance component. This section is used in obtaining feasible sets on red and blue channel where high frequency obtained is similar to the frequency of the green channel.

Reconstruction process is provide by decompose the feasible sets by using two filters $\mathrm{H}_{0}(\mathrm{z})$ and $\mathrm{H}_{1}(\mathrm{z})$. These two filters provide better reconstruction process with synthesis filter $\mathrm{S}_{0}(\mathrm{z})$ and $\mathrm{S}_{1}(\mathrm{z})$.

It can be written as $H_{0}(z) S_{0}(z)+H_{1}(z) S_{1}(z)=1$

Let $\mathrm{h}_{0}(). \& \mathrm{~h}_{1}($.$) denote the impulse response of \mathrm{H}_{0}(\mathrm{z}) \& \mathrm{H}_{1}(\mathrm{z})$. we can write four sub bands with two dimensional signal $\mathrm{X}\left(\mathrm{t}_{1}, \mathrm{t}_{2}\right)$ as follows.

$\mathrm{W}_{1}=\left[\mathrm{h}_{0}\left(\mathrm{t}_{1}\right) * \mathrm{~h}_{0}\left(\mathrm{t}_{2}\right)\right] * \mathrm{X}\left(\mathrm{t}_{1}, \mathrm{t}_{2}\right)$

$\mathrm{W}_{2}=\left[\mathrm{h}_{1}\left(\mathrm{t}_{1}\right) * \mathrm{~h}_{0}\left(\mathrm{t}_{2}\right)\right] * \mathrm{X}\left(\mathrm{t}_{1}, \mathrm{t}_{2}\right)$

$\mathrm{W}_{3}=\left[\mathrm{h}_{0}\left(\mathrm{t}_{1}\right) * \mathrm{~h}_{1}\left(\mathrm{t}_{2}\right)\right] * \mathrm{X}\left(\mathrm{t}_{1}, \mathrm{t}_{2}\right)$

$\mathrm{W}_{4}=\left[\mathrm{h}_{1}\left(\mathrm{t}_{1}\right) * \mathrm{~h}_{1}\left(\mathrm{t}_{2}\right)\right] * \mathrm{X}\left(\mathrm{t}_{1}, \mathrm{t}_{2}\right)$

By denoting $\mathrm{S}_{0}$ (.) \& $\mathrm{S}_{1}\left(\right.$.) as the impulse response of $\mathrm{S}_{0}(\mathrm{z}) \&$ $\mathrm{S}_{1}(\mathrm{z})$ then the filtering operations on a two level dimensional signal as follows

$\mathrm{Y}_{1}=\left[\mathrm{S}_{0}\left(\mathrm{t}_{1}\right) * \mathrm{~S}_{0}\left(\mathrm{t}_{2}\right)\right] * \mathrm{X}\left(\mathrm{t}_{1}, \mathrm{t}_{2}\right)$

$\mathrm{Y}_{2}=\left[\mathrm{S}_{1}\left(\mathrm{t}_{1}\right) * \mathrm{~S}_{0}\left(\mathrm{t}_{2}\right)\right] * \mathrm{X}\left(\mathrm{t}_{1}, \mathrm{t}_{2}\right)$

$\mathrm{Y}_{3}=\left[\mathrm{S}_{0}\left(\mathrm{t}_{1}\right) * \mathrm{~S}_{1}\left(\mathrm{t}_{2}\right)\right] * \mathrm{X}\left(\mathrm{t}_{1}, \mathrm{t}_{2}\right)$

$\mathrm{Y}_{4}=\left[\mathrm{S}_{1}\left(\mathrm{t}_{1}\right) * \mathrm{~S}_{1}\left(\mathrm{t}_{2}\right)\right] * \mathrm{X}\left(\mathrm{t}_{1}, \mathrm{t}_{2}\right)$
Where $\mathrm{Y}_{1}, \mathrm{Y}_{2}, \mathrm{Y}_{3}, \mathrm{Y}_{4}$ are synthesis filter operators. This form a reconstruction filter bank with approximate sub bands $\mathrm{W}_{1}, \mathrm{~W}_{2}, \mathrm{~W}_{3}, \mathrm{~W}_{4}$.

$\mathrm{X}\left(\mathrm{t}_{1}, \mathrm{t}_{2}\right)=\mathrm{Y}_{1} \mathrm{~W}_{1}+\mathrm{Y}_{2} \mathrm{~W}_{2}+\mathrm{Y}_{3} \mathrm{~W}_{3}+\mathrm{Y}_{4} \mathrm{~W}_{4}$

Now we define detail feasible set $F_{d}$ as follows.

$\mathrm{F}_{\mathrm{d}}=\left\{\mathrm{X}\left(\mathrm{t}_{1}, \mathrm{t}_{2}\right):\left|\left(\mathrm{W}_{1}\right)\left(\mathrm{t}_{1}, \mathrm{t}_{2}\right)-\left(\mathrm{W}_{\mathrm{k}}\right)\left(\mathrm{t}_{1}, \mathrm{t}_{2}\right)\right| \leq \mathrm{T}\left(\mathrm{t}_{1}, \mathrm{t}_{2}\right)\right.$

for $\mathrm{K}=2,3,4 \mathrm{X}=\mathrm{R}, \mathrm{B}$

It enhances the high frequency component of red and blue channel to be similar to green channel. Here $\mathrm{T}$ is the threshold which defines the color channel correlation. If the color channels are highly correlated, threshold gets to be small. If the color channel is not correlated threshold become larger. In order to set $\mathrm{T}$ to zero, high correlation of color channel is required.

7.1 Projection operator: The projection $\mathrm{P}_{0}$ for observation constraint set is obtained from the feasible set given in (6). From that equation, we can write $\mathrm{P}_{0}$ onto "observation" feasible set as follows:

$\mathrm{P}_{0}\left[\mathrm{X}\left(\mathrm{t}_{1}, \mathrm{t}_{2}\right)\right]=\left\{\begin{array}{c}D\left(t_{1}, t_{2}\right), \epsilon \wedge s \\ X\left(t_{1}, t_{2}\right), \text { otherwise }\end{array}\right.$

The projection $\mathrm{P}_{\mathrm{d}}$ for detail feasible set $\mathrm{F}_{\mathrm{d}}$ is given as

$\mathrm{P}_{\mathrm{d}}\left[\mathrm{x}\left(\mathrm{t}_{1}, \mathrm{t}_{2}\right)\right]=\mathrm{Y}_{1}\left(\mathrm{~W}_{1}\right)\left(\mathrm{t}_{1}, \mathrm{t}_{2}\right)+\sum_{\mathrm{k}=2}^{4} \mathrm{Y}_{\mathrm{k}}\left(\mathrm{W}_{\mathrm{k}}\right)\left(\mathrm{t}_{1}, \mathrm{t}_{2}\right)$

\section{SIMULATION RESULTS}

Simulation was carried out to estimate the performance of the proposed algorithm. The 24 digital color images shown in Fig. 5 were used to generate a set of testing images. The peak signal-to-noise ratio (PSNR) was used as a measure to express the performance of the demosaicing methods. In PSNR, particular PSNR for each color sample is defined as

$$
C P S N R=10 \log _{10}\left(\frac{255^{2}}{M S E}\right)
$$

Where,

$$
M S E=\sum_{i, j} \frac{\left(I_{o r g}(i, j)-I_{r}(i, j)\right)^{2}}{m * n}
$$

$I_{\text {org }}$-represent the original image of size $m * n$

$I_{r}$-represent the reconstructed image of size $m * n$

Images are numbered from 1 to 24 in the order of left-to-right and top-to-bottom was used in the experiments 


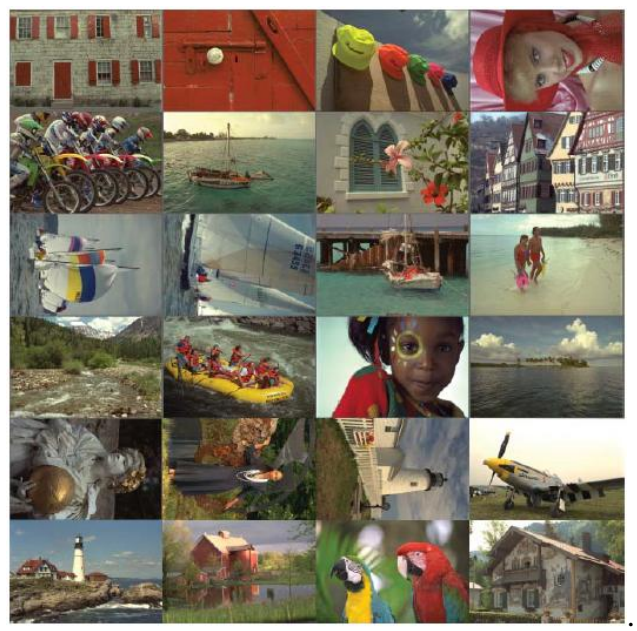

Fig 6[11]: Images used for Simulation

Table- 1: Compare the proposed method with existing demosaicing algorithm

\begin{tabular}{|c|c|c|c|c|c|}
\hline $\begin{array}{c}\mathrm{Im} \\
\mathrm{agg} \\
\mathrm{e}\end{array}$ & Bilinear & $\begin{array}{l}\text { Edge } \\
\text { based }\end{array}$ & $\begin{array}{l}\text { Subband } \\
\text { synthesi } \\
\text { s }\end{array}$ & $\begin{array}{l}\text { Wavelet } \\
\text { with } \\
\text { spatial } \\
\text { refinemen } \\
\mathrm{t}\end{array}$ & $\begin{array}{l}\text { Proposed } \\
\text { method }\end{array}$ \\
\hline 1 & 30.773 & 31.2458 & 39.5224 & 40.6942 & 42.8064 \\
\hline 2 & 36.6873 & 37.0425 & 39.5198 & 41.3527 & 45.6784 \\
\hline 3 & 37.6775 & 38.0647 & 43.3218 & 43.9651 & 47.7373 \\
\hline 4 & 37.4213 & 37.8047 & 41.4224 & 43.4641 & 46.6931 \\
\hline 5 & 31.2157 & 31.8233 & 38.6369 & 40.5972 & 43.5113 \\
\hline 6 & 32.1414 & 32.5964 & 40.255 & 41.9364 & 44.1239 \\
\hline 7 & 37.1797 & 37.6152 & 42.1956 & 44.6154 & 46.7256 \\
\hline 8 & 28.2879 & 28.7457 & 36.0158 & 38.9305 & 42.0271 \\
\hline 9 & 36.4896 & 36.9292 & 43.2609 & 45.2354 & 47.4915 \\
\hline 10 & 36.3952 & 36.8716 & 43.5337 & 45.2596 & 47.8490 \\
\hline 11 & 33.6645 & 34.1372 & 41.006 & 42.9933 & 44.0718 \\
\hline 12 & 36.9695 & 37.3912 & 43.5063 & 46.4256 & 49.6349 \\
\hline 13 & 28.6113 & 29.1087 & 37.2893 & 37.3747 & 41.9504 \\
\hline 14 & 33.469 & 33.9611 & 36.7377 & 38.7378 & 44.0018 \\
\hline 15 & 35.4486 & 35.8213 & 39.8739 & 42.0606 & 46.1802 \\
\hline 16 & 35.2366 & 35.6156 & 43.2864 & 44.6285 & 44.5141 \\
\hline 17 & 36.681 & 37.2463 & 43.6912 & 44.4829 & 46.8972 \\
\hline 18 & 32.562 & 33.0611 & 38.9911 & 40.0813 & 43.8988 \\
\hline 19 & 32.7829 & 33.2797 & 40.5543 & 43.9703 & 45.6252 \\
\hline
\end{tabular}

\begin{tabular}{|l|c|c|c|c|c|}
\hline 20 & 34.6422 & 34.9845 & 41.2988 & 42.7135 & 47.6793 \\
\hline 21 & 33.0627 & 33.5363 & 40.9836 & 41.8151 & 45.1638 \\
\hline 22 & 34.9277 & 35.3907 & 39.492 & 40.9682 & 45.3023 \\
\hline 23 & 38.3544 & 38.7374 & 42.7698 & 43.9694 & 48.5216 \\
\hline 24 & 31.411 & 31.8746 & 37.6036 & 38.0094 & 45.2865 \\
\hline
\end{tabular}

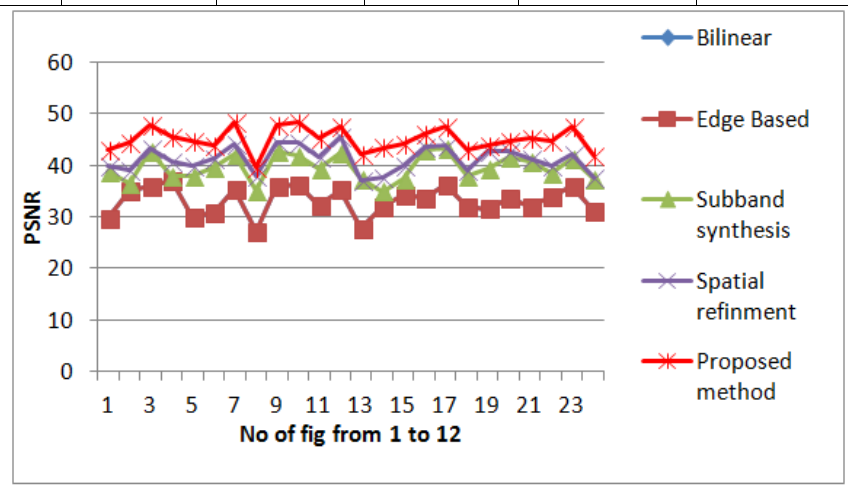

Fig 7 PSNR value comparisons of red channel with existing method

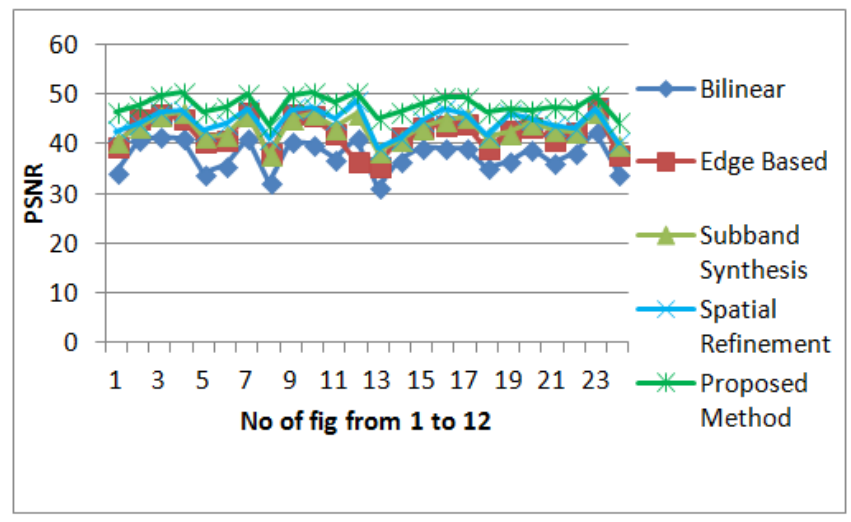

Fig 8: PSNR value comparisons of green channel with existing method

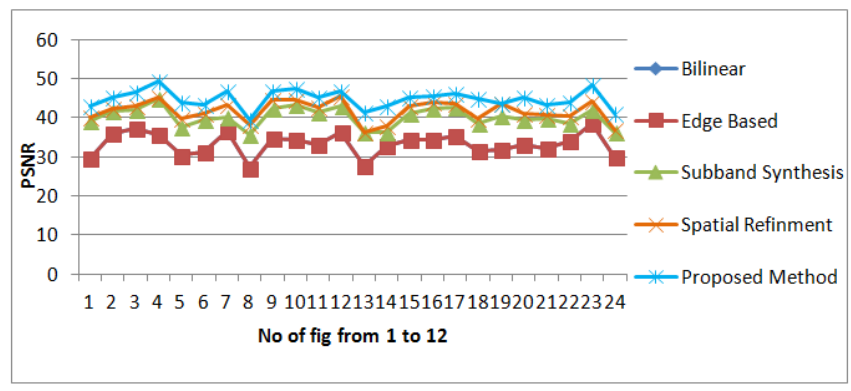

Fig 9: PSNR value comparisons of blue channel with existing demosaicing algorithms 
Table -2: Compare the CPSNR value of proposed method with existing method

\begin{tabular}{|c|c|c|c|c|c|c|c|c|c|c|c|c|c|c|c|}
\hline \multirow[t]{2}{*}{ Img } & \multicolumn{3}{|c|}{ Bilinear } & \multicolumn{3}{|c|}{ Edge based } & \multicolumn{3}{|c|}{ Subband synthesis } & \multicolumn{3}{|c|}{$\begin{array}{c}\text { Wavelet with spatial } \\
\text { refinement }\end{array}$} & \multicolumn{3}{|c|}{ Proposed method } \\
\hline & $\mathrm{R}$ & G & B & $\mathrm{R}$ & G & B & $\mathrm{R}$ & G & B & $\mathrm{R}$ & G & B & $\mathrm{R}$ & G & B \\
\hline 1 & 29.741 & 34.286 & 29.678 & 29.741 & 39.442 & 29.678 & 38.881 & 40.591 & 39.272 & 39.811 & 42.617 & 40.154 & 43.1701 & 46.5192 & 43.0099 \\
\hline 2 & 35.022 & 40.797 & 36.100 & 35.022 & 44.854 & 36.100 & 36.524 & 43.533 & 41.665 & 39.147 & 44.034 & 42.327 & 44.5201 & 47.8272 & 45.1250 \\
\hline 3 & 35.942 & 41.591 & 37.252 & 35.942 & 45.912 & 37.252 & 42.825 & 45.853 & 42.113 & 43.217 & 46.289 & 43.078 & 47.7906 & 49.7287 & 46.5177 \\
\hline 4 & 37.106 & 41.126 & 35.663 & 37.106 & 45.051 & 35.663 & 37.928 & 46.396 & 44.927 & 40.751 & 46.799 & 45.211 & 45.4878 & 50.4066 & 49.4103 \\
\hline 5 & 30.126 & 34.082 & 30.430 & 30.126 & 40.240 & 30.430 & 37.853 & 41.500 & 37.547 & 39.959 & 42.717 & 39.705 & 44.6659 & 46.5055 & 43.8606 \\
\hline 6 & 30.803 & 35.699 & 31.355 & 30.803 & 40.606 & 31.355 & 39.616 & 41.921 & 39.609 & 41.342 & 44.180 & 40.943 & 43.9950 & 47.5484 & 43.3552 \\
\hline 7 & 35.514 & 41.092 & 36.662 & 35.514 & 46.389 & 36.662 & 42.159 & 45.984 & 40.226 & 44.156 & 47.043 & 43.420 & 48.4452 & 50.0155 & 46.7127 \\
\hline 8 & 27.104 & 32.181 & 27.220 & 27.104 & 37.959 & 27.220 & 34.998 & 37.820 & 35.697 & 37.959 & 41.183 & 38.307 & 39.6434 & 43.6341 & 39.5488 \\
\hline 9 & 36.102 & 40.438 & 34.717 & 36.102 & 45.917 & 34.717 & 42.785 & 45.225 & 42.298 & 44.552 & 46.919 & 44.624 & 47.7729 & 49.7786 & 46.7624 \\
\hline 10 & 36.272 & 40.017 & 34.528 & 36.272 & 45.509 & 34.528 & 41.980 & 46.187 & 43.416 & 44.351 & 47.456 & 44.598 & 48.4588 & 50.3640 & 47.3625 \\
\hline 11 & 32.269 & 37.035 & 33.016 & 32.269 & 41.880 & 33.016 & 39.313 & 43.338 & 41.279 & 41.738 & 45.071 & 42.801 & 45.2345 & 48.5037 & 45.2789 \\
\hline 12 & 35.266 & 41.164 & 36.404 & 35.266 & 36.404 & 36.404 & 42.376 & 46.138 & 42.872 & 45.619 & 48.716 & 45.612 & 47.5609 & 50.4384 & 46.9416 \\
\hline 13 & 27.650 & 31.312 & 27.764 & 27.650 & 35.342 & 27.764 & 37.443 & 38.584 & 36.173 & 37.128 & 39.053 & 36.363 & 42.1709 & 45.0075 & 41.2764 \\
\hline 14 & 32.123 & 36.675 & 32.828 & 32.123 & 41.524 & 32.828 & 34.990 & 40.838 & 36.262 & 37.767 & 41.174 & 38.021 & 43.4756 & 46.3178 & 43.0135 \\
\hline 15 & 34.216 & 39.315 & 34.439 & 34.216 & 43.306 & 34.439 & 37.339 & 43.381 & 41.055 & 39.927 & 44.670 & 42.936 & 44.2827 & 47.9899 & 45.1903 \\
\hline 16 & 33.815 & 39.277 & 34.378 & 33.815 & 43.662 & 34.378 & 43.143 & 44.836 & 42.260 & 43.632 & 46.928 & 44.008 & 46.2854 & 49.4875 & 45.5253 \\
\hline 17 & 36.205 & 39.248 & 35.440 & 36.205 & 43.949 & 35.440 & 43.318 & 45.433 & 42.754 & 43.964 & 46.035 & 43.786 & 47.4677 & 49.3932 & 46.1630 \\
\hline 18 & 31.906 & 35.128 & 31.478 & 31.906 & 38.980 & 31.478 & 37.865 & 41.464 & 38.428 & 39.039 & 41.776 & 39.860 & 43.1096 & 46.4512 & 44.9765 \\
\hline 19 & 31.592 & 36.521 & 31.772 & 31.592 & 42.845 & 31.772 & 39.476 & 42.313 & 40.337 & 42.768 & 45.944 & 43.773 & 43.9892 & 47.0844 & 43.6487 \\
\hline 20 & 33.838 & 39.032 & 33.080 & 33.838 & 43.287 & 33.080 & 41.613 & 44.279 & 39.349 & 42.673 & 45.111 & 41.206 & 44.7645 & 46.6920 & 45.2220 \\
\hline 21 & 31.892 & 36.279 & 32.221 & 31.892 & 40.838 & 32.221 & 40.720 & 42.780 & 39.923 & 41.446 & 43.773 & 40.762 & 45.1942 & 47.4017 & 43.4365 \\
\hline 22 & 33.866 & 38.111 & 33.983 & 33.866 & 42.446 & 33.983 & 38.562 & 42.531 & 38.472 & 39.964 & 43.059 & 40.467 & 44.7391 & 47.0056 & 43.9862 \\
\hline 23 & 36.114 & 42.556 & 38.598 & 36.114 & 47.324 & 38.598 & 41.226 & 46.550 & 42.145 & 42.076 & 47.010 & 44.159 & 47.5222 & 49.6431 & 48.4389 \\
\hline 24 & 31.062 & 34.104 & 30.016 & 31.062 & 37.717 & 30.016 & 37.292 & 40.023 & 36.291 & 37.664 & 40.261 & 36.797 & 42.0336 & 44.3045 & 41.1282 \\
\hline
\end{tabular}

From Table 2, it is acknowledged that bilinear interpolation method fails to detect the edge in images. In edge based method as shown in Table 3. having high CPSNR in G but not determine edges in $\mathrm{R}$ and $\mathrm{B}$. As compare to edge based method, sub band synthesis having high PSNR but it cause color artifacts in images due to high frequency component. In spatial refinement process, zipper effect cause due to longer filters. However in proposed method, based on projection algorithm and gradient based interpolation method weight is determined for every pixel and refinement take place before and after interpolating of image. By comparing the performance of various algorithms, the proposed method gives better performance among the existing methods.

\section{CONCLUSIONS}

In this paper, Color Filter Array based on HVS and alternative demosaicing algorithm with enhanced border and edge was presented. It makes use of the color difference variance of the pixels located along the four directions in a local region to estimate the interpolation direction for interpolating the missing green samples. The highperformance arises from the introduction of a gradient based interpolation method. With them, the proposed algorithm has a good initial condition and can terminate algorithm early. The main advantage of the proposed interpolation is its computational efficiency for green. Simulation results 
show that the proposed method is able to process a subjectively and objectively best demosaicing results

\section{REFERENCES}

[1] Parulski, K. and K. E. Spaulding, 2002. Color image processing for digital cameras, in Digital Color Imaging Handbook, (eds.) G. Sharma, CRC Press, Boca Raton, FL., 2 :728-757.

[2] Dillon, P.L.P., D. M. Lewis, and F. G. Kaspar, 1978. Color imaging system using a single CCD area array, IEEE Journal of Solid-State Circuits, 13(1) : 28-33.

[3] Lule, T., S.Benthien, and H. Keller,et al., 2000. Sensitivity of CMOS based imagers and scaling perspectives, IEEE Transactions on Electron Devices, 47( 11) : 2110-2122.

[4] Lukac, R. and K. N. Plataniotis, 2005. Data-adaptive filters for demosaicking: A framework, IEEE Transactions on Consumer Electronics,51(2):560-570.

[5] Technical Committee ISO/TC 42, 2001. Photography, Electronic still picture imaging - Removable memory, Part 2: Image data format - TIFF/EP, ISO 12234-2.

[6] Lukac, R. and K. N. Plataniotis, 2005. Fast video demosaicking solution for mobile phone imaging applications, IEEE Transactions on Consumer Electronics, 51(2): 675-681.

[7] Wu, X. and N. Zhang, 2004. Primary-consistent softdecision color demosaicking for digital cameras, IEEE Transactions on Image Processing,13(9):1263-1274.

[8] Lukac, R., B. Smolka, K. Martin, K. N. Plataniotis, and A. N.Venetsanopulos, 2005. Vector filtering for color imaging, IEEE Signal Processing Magazine;22(1):74-86.

[9] Bayer, B.E., 1976. Color imaging array, U.S. Patent 3971 065 .

[10] Haddad, R.A., and A.N. Akansu, 1991. A Class of Fast Gaussian Binomial Filters for Speech and Image Processing, IEEE Transactions on Acoustics, Speech and Signal Processing,39:723-727.

[11] Gunturk, B.K. ,Y.Altunbasak and R. M. Mersereau, 2002. Color plane interpolation using alternating projections, IEEE Trans.on Image Process, 11(9): 997-1013.

[12] Parmar, M. and S.J. Reeves, 2004. A perceptually based design methodology for color filter arrays, in Proc.IEEE Int. Con. Acoustics, Speech, and Signal Processing (ICASSP'04), 3: 473- 476.

[13] Hirakawa, K. and T.W.Parks, 2005. Adaptive homogeneity-directed demosaicing algorithm, IEEE Trans .on Image Process, 141(39): 360-369.

[14] Alleysson, D., S. Süsstrunk, and J. Hérault,2005. Linear demosaicing inspired by the human visual system, IEEE Trans. Image Process, 14(4): 439-449.
[15] Haddad, R.A. and A.N. Akansu,1991. A Class of Fast Gaussian Binomial Filters for Speech and Image Processing, IEEE Transactions on Acoustics, Speech and Signal Processing,39: 723-727.

[16] Kakarala, R. and Z. Baharav, 2002. Adaptive demosaicing with the principal vector method, IEEE Trans. Consum. Electron.,48(11):932-937.

[17] king-Hong Chung,. and Yuk-Hee Chan, 2006. Color demosaicing using variance of color differences, IEEE Transactions on Image Processing, 15(10):2944-2955. 